\title{
Editorial
}

\author{
Complementary \\ Medicine Research \\ Practice|Methods|Perspectives
}

Complement Med Res 2019;26:289-292

DOI: 10.1159/000503909
Received: October 4, 2019

Accepted: October 7, 2019

Published online: October 8, 2019

\section{Neues aus der Veterinärmedizin}

\author{
Michael Walkenhorst \\ Departement für Nutztierwissenschaften, Forschungsinstitut für biologischen Landbau FiBL, Frick, Schweiz
}

Knapp 2 Jahre sind mittlerweile vergangen seit dem letzten "tierischen" Editorial [1], höchste Zeit für eine Bestandsaufnahme und ein Update. Ein halbes Dutzend veterinärmedizinisch relevante Publikationen sind in den vergangenen rund zwei Jahren in Complementary Medicine Research (CMR) erschienen [2-7], zwei weitere, so viel sei an dieser Stelle schon verraten, befinden sich aktuell im Review-Prozess. Auch zwei Journal Clubs und Abstract Services [8-11] waren tiermedizinisch ausgerichtet. Dies ist ein guter Anfang, aber durchaus noch ausbaufähig. Dies insbesondere vor dem Hintergrund, dass mittlerweile die tierärztlichen Mitglieder der SMGP die 100er-Latte und gleichzeitig auch deutlich die 10\%Marke gerissen haben. Es wäre erstrebenswert, wenn zukünftig ein vergleichbarer Prozentsatz an Artikeln der CMR auch explizit von tierärztlichem Interesse wären. Mit dieser "Vision" möchte ich gerne mein Editorial eröffnen und auch gleich alle Leserinnen und Leser motivieren, entsprechende Manuskripte einzureichen. Dies dürfen zum Beispiel auch gut dokumentierte interessante Fallbeispiele oder auch retrospektiv aufbereitete Fallserien aus der eigenen Praxis sein. Während beispielsweise das landwirtschaftliche Erfahrungswissen zum Einsatz von Arzneipflanzen bei landwirtschaftlichen Nutztieren in der Schweiz mittlerweile gut dokumentiert ist $[5,12$, 13], böte die hochwertige Dokumentation tierärztlicher Erfahrungen mit Arzneipflanzen (insbesondere bei Pferden und Kleintieren) noch üppig Raum.

Weitere Anregung für Forschungsideen rund um Pflanzen im Einsatz für die Tiergesundheit eröffnet ein Blick in die Programme des Kongresses der Gesellschaft für Arzneipflanzen- und Naturstoffforschung, der vom 1.-4. September 2019 in Innsbruck in Österreich stattfand (www.ga2019.at/pre-congress-symposia/animalhealthcare-and-veterinary-phytotherapy.html), der Jah- restagung der GPT vom 19.-21 September 2019 in Münster in Deutschland (https://phytotherapie.de/de/ termine/phytotherapie-20191/programm/) oder natürlich ganz besonders unserer 34. Jahrestagung für Phytotherapie in Baden in der Schweiz am 21. November 2019 (https://phytotherapie.de/de/termine/phytotherapie-20191/programm/). Das diesjährige Motto des Parallelsymposiums Veterinärmedizin: "Eine Schnauzenlänge voraus - Veterinärphytotherapie rund um den Kopf".

Die Tiermedizin kommt also in der Phytotherapie an und die Phytotherapie in der Tiermedizin. Ein weiteres wichtiges Indiz hierfür ist eine Webseite: Im deutschsprachigen Raum dürfte kaum einem Tierarzt, kaum einer Tierärztin die "CliniPharm CliniTox" (www.tierarzneimittel.ch) Webseite unbekannt sein. Dieses vom Institut für Veterinärpharmakologie und -toxikologie der Universität Zürich herausgegebene und von beiden veterinärpharmakologisch und -toxikologischen Instituten der Schweiz (Bern und Zürich) mitverantwortete - nach wie vor frei und kostenlos (!) verfügbare - ausserordentlich umfangreiche Informationssystem zu allen Fragen rund um den Medikamenten- und Wirkstoffeinsatz sowie das Giftstoffrisiko bei Tieren hat unlängst die Kategorie "Arzneipflanzen" eröffnet (www.phytoarznei.ch) [14]. Ähnlich humanmedizinischer Monographien sind die Informationen für jede Pflanzendroge in Rubriken kategorisiert. "Verwendete Pflanzenteile", "Pharmakognosie" (organoleptische Angaben, Inhaltsstoffe) und "Pharmakologie" stellen einen Überblick zu allgemeinen Informationen zu den entsprechenden Drogen dar. In der Rubrik Veterinärmedizin "werden anerkannte und traditionelle tiermedizinische Anwendungen sowie ethnoveterinärmedizinische Studien, Zubereitung, Dosierungsempfehlung, besondere Hinweise, Toxizität, Fertigpräparate, gesetzliche Vorschriften und Anmerkungen zu Doping auf-

\section{KARGER}

(C) 2019 S. Karger AG, Basel 
geführt" [14]. Auch die Rubrik Humanmedizin ist für einige der aktuell mittlerweile über 60 Drogen bereits abgebildet - reinschauen lohnt sich!

Das freundliche, engagierte und beherzte Öffnen der Türen der beiden schweizerischen veterinärpharmakologischen Institute für die Veterinärphytotherapie ist einzigartig und für die Anwendung von Arzneipflanzen bei Tieren in Europa von unschätzbarem Wert. Ein ausdrücklicher Dank an die beiden für diese Öffnung verantwortlichen Personen Meike Mevissen (Direktorin der Abteilung für Veterinär-Pharmakologie und Toxikologie in Bern) und Hanspeter Nägeli (Direktor des Instituts für Veterinärpharmakologie und -toxikologie in Zürich) sei an dieser Stelle erlaubt und auch und besonders an Jacqueline Kupper, die treibende Kraft hinter dieser Webseite. Gleichsam bringt dies aber auch eine hohe Verantwortung: Mit Nachdruck weiterhin qualitativ hochwertige Forschung zum Einsatz von Arzneipflanzen beim Tier zu betreiben und, Sie ahnen schon was nun kommt, wissenschaftlich zu publizieren, ist unverzichtbare Basis für den Ausbau dieses Informationssystems.

\section{Ist die Phytotherapie so alt wie die Menschheit?}

Dies lässt sich aus heutiger Sicht gleich zweifach verneinen, zumindest falls wir die Menschheit über das Auftreten des Homo sapiens definieren. Es spricht einiges dafür, dass schon der Neandertaler auf Arzneipflanzen gesetzt hat, wie die Verteilung zwischen rein nutritiven Pflanzen und solchen mit medizinischen Möglichkeiten wahrscheinlich erscheinen lässt [15]. Das zweite Nein lässt sich aus der Forschung zur Selbstmedikation von Tieren der vergangenen 30 Jahre ableiten $[16,17]$. Die Phytotherapie ist folglich so alt wie die "Tierheit" (wobei diese im Gegensatz zur Menschheit ja eine überaus grosse Zahl an Spezies umfasst). Selbst von Schmetterlingen ist eine gewisse Form der Selbstmedikation bekannt [18] Nicht zuletzt deutet einiges darauf hin, dass Menschen den Einsatz von Arzneipflanzen mitunter auch von Tieren gelernt haben [16].

Wie lässt es sich aber erklären, dass Tiere gelernt haben sich selbst zu medizinieren? Denken Sie einmal an Ihre letzte Magen-Darm-Grippe und (bitte entschuldigen Sie diesen nun folgenden möglicherweise im wahrsten Sinne des Wortes üblen Gedanken, aber in einem medizinischen Journal sei dieser virtuelle Selbstversuch erlaubt) nun anschliessend an die unmittelbar zuvor eingenommenen Mahlzeit. Ich selbst (und wer mich kennt, weiss, dass ich wahrhaft nicht zu den Kostverächtern, sondern eher zum Typ "Allesfresser" gehöre) konnte nach einem entsprechenden "Erlebnis" über Monate heisse Brühwürstchen nicht einmal mehr riechen ohne ein flaues Gefühl in der Magengrube. Die Würstchen wa- ren allerdings mit grosser Wahrscheinlichkeit nicht die Auslöser der Symptomatik, dem Rest der mitessenden Gesellschaft sind sie wohlbekommen. Es waren also nicht den Würstchen innenwohnende Toxine, die meine Aversion ausgelöst hatten, sondern ein Geruchs- und Geschmackserlebnis, dass sich mit einer (in diesem Falle als negativ empfundenen) pathophysiologischen Aktion meines Körpers verknüpfte, die mit der Zeit allerdings auch wieder in Vergessenheit geriet, da sich dieses Erlebnis zum Glück nicht wiederholte. Entsprechende Rückkoppelung scheint es zum Teil wesentlich feinfühliger und vor allem auch in positiver Hinsicht bei Tieren $\mathrm{zu}$ geben. Beispielhaft seien an dieser Stelle zwei einfache, jedoch in ihrer Einfachheit gleichsam geniale Versuche aus der Arbeitsgruppe rund um Fred Provenza und Juan Villalba von der Utah State University dargestellt:

Ein erstes Experiment: Die reine Fütterung von Stroh führt bei Schafen zu einem Energiedefizit - Schafe werden von Stroh nicht satt. Zunächst wurden nun zwei unterschiedlich aromatisierte Strohsorten hergestellt - ein Stroh mit Apfelgeschmack und ein weiteres mit Ahornsirupgeschmack. Nach dem Verzehr des Strohs mit Apfelgeschmack wurde der einen Gruppe eine energiereiche Flüssignahrung, der anderen Gruppe im selben Volumen Wasser verabreicht. Im Anschluss wurde auf Stroh mit Ahornsirupgeschmack gewechselt und die Schafe, die nach dem Stroh mit Apfelgeschmack die Flüssignahrung erhielten, bekamen nun Wasser und die andere Gruppe die Flüssignahrung. Das wurde einige Zeit so fortgeführt und anschliessend ein Wahlversuch mit beiden Strohsorten durchgeführt mit dem Ergebnis, dass sich die Schafe heisshungrig zielgenau auf jeweils das Stroh stürzten, wonach sie die Flüssignahrung erhielten; das jeweils anderweitig aromatisierte Stroh wurde verschmäht [19].

Ein zweites Experiment: Das Aufblähen kommt bei Wiederkäuern immer wieder vor und dürfte sicher als negativ empfunden werden. Schafen wurde ein Ballon in den Pansen eingebracht, den man aufblasen oder auch wieder entspannen konnte. Einer Gruppe von Schafen bot man für eine Viertelstunde Weizenkleie an, die sie auch frassen, und blies anschliessend den Ballon für eine halbe Stunde lang auf. Daraufhin bekamen die Schafe Zuckerrübenschnitzel und der Ballon wurde kurz darauf wieder abgelassen. Eine zweite Gruppe von Schafen erhielt die beiden Futtermittel bei ansonsten gleichem Versuchsdesign in umgekehrter Reihenfolge. Zwei weitere Gruppen von Schafen wurden nicht künstlich aufgebläht, aber sie erhielten die beiden Futtermittel in jeweils entsprechender Reihenfolge wie die beiden zuvor beschriebenen Versuchsgruppen angeboten. Nach dieser Erfahrung reagierten im Vergleich zu den "unaufgeblähten" Vergleichsgruppen die Schafe, denen der Pansen gebläht wurde, nicht nur mit einer klaren Aversion gegenüber 
dem "blähenden" Futter sondern, und das war das eigentlich Erstaunliche, mit einer sehr deutlichen Bevorzugung jeweils des Futters, nach dessen Verzehr das wohltuende Entblähen stattfand [20].

Tiere sind in der Lage, sensorische Wahrnehmungen mit darauf (durchaus auch zeitverzögert) folgenden Reaktionen ihres Körpers zu verknüpfen - sowohl mit positiven als auch mit negativen. Provenza und Kollegen sprechen von "biochemically mediated flavor-feedback" [17].

Erwähnenswert ist an dieser Stelle auch noch ein drittes Ergebnis aus der Arbeitsgruppe: Ganz offensichtlich zeigen unsere domestizierten Wiederkäuer tageszeitlich unterschiedliche Präferenzen für einzelne Futterpflanzen. Sie frühstücken, essen zu Mittag und zu Abend und nehmen auch die eine oder andere Zwischenmahlzeit ein und das durchaus sehr individuell unterschiedlich [21]. Welche Einschränkung bedeutet vor diesem Hintergrund für Milchkühe die "moderne" Fütterung? Sie besteht nur noch aus wenigen Pflanzenarten (Mais, einige wenige Gräserarten, Rot-, Weissklee und Luzerne sowie Getreide und Körnerleguminosen) und wird als gutdurchmischter "Einheitsbrei" ("Totalmischration") angeboten.

Einen anderen Aspekt des "flavor-feedback" macht sich die Tiermedizin aktuell zu Nutze, wie dies die Tierärztin und Verhaltenstherapeutin Daniela Zurr am vergangenen Kurs 7 der SMGP Ende September 2019 eindrucksvoll aus eigener Praxis berichtete. Verhaltensauffällige Hunde werden unter Zuhilfenahme von Gerüchen, z.B. auf Basis ätherischer Öle (wohlbemerkt ausschliesslich solcher, die der individuelle Hund zuvor im Test als "interessant" identifiziert hatte), darauf trainiert, Angstsituationen (z.B. vor Gewittern oder Silvesterknallern) besser bewältigen zu können.

Beschränkt sich die sensorische Wahrnehmung ausschliesslich auf riechen und schmecken? Lange Jahre ging man davon aus, Riechrezeptoren gehören in die Nase, Geschmacksrezeptoren auf die Zunge, Punkt. Doch diese Zeiten sind vorbei. Entsprechende Rezeptoren findet man mittlerweile in nahezu allen Körpergeweben des Menschen. Auch wenn bereits die diagnostische und therapeutische Bedeutung dieser Rezeptoren intensiv diskutiert wird, steckt die Forschung über die zahlreichen Effekte, die die Anregung dieser Sensoren beim Menschen bewirken, noch in den Kinderschuhen [22]. Hinsichtlich der Nutz- und Heimtiere dürfte sie noch weniger weit sein. Chemorezeptoren, zu denen die Riechrezeptoren zweifelsohne gehören, sind überdies nicht den Eukaryoten vorbehalten; auch Bakterien haben solche [23]. Man könnte also auch diesen schon zubilligen, "riechen" und "schmecken" zu können und "Zu-" und "Abneigungen" zu entwickeln. Der "biochemically mediated flavor-feedback" [17] könnte noch sehr viel weitreichender als bisher relevant sein.

Neues aus der Veterinärmedizin
Fassen wir zusammen: Mensch und Tier sind offensichtlich dazu in der Lage, Sinneseindrücke (überwiegend aus Mund/Maul und Nase) mit einer sich anschliessenden Befindensänderung zu verknüpfen. Wird diese Befindensänderung (anders als in den vorgestellten Versuchen, aber nah an der Realität der Lebensbedingungen von Tieren, insbesondere von Wildtieren) unmittelbar von der Pflanze ausgelöst, die sie gefressen haben, lernen sie, Pflanzen gezielt für sich und ihr Wohlbefinden einzusetzen.

Teile dieses Erlernten geben sie an ihre Nachkommen weiter [19]. Das Selektionsverhalten von individuellen Tieren, auch das selbst-medizinierende, setzt sich folglich aus einem Teil von den Eltern und einem Teil aus selbst neu Erlerntem zusammen. Um die eingangs gestellte Frage nun nochmals, zugegeben etwas kühn, zu beantworten: Nein! Schon weit vor dem Menschen haben Tiere die Phytotherapie entdeckt, und das sogar bereits ansatzweise im Sinne einer umfassenden Definition der individualisierten Medizin [24], und Tiere (und vielleicht sogar auch ein paar Menschen) entdecken sie tagtäglich neu.

\section{Neues aus dem Tierarzneimittelrecht}

Apropos neu: Was hat sich zwischenzeitlich hinsichtlich der Rechtslage für die Neuzulassung phytotherapeutischer Tierarzneimittel getan? Da gibt es einerseits sehr Erfreuliches und andererseits zumindest von einer gewissen Erleichterung zu berichten. Zunächst die Erleichterung: Es stand einige Zeit auf der Kippe, was im Zuge der neuen EU-Tierarzneimittelverordnung mit den Arzneipflanzen passieren würde. Im Gegensatz zur Homöopathie, deren Einsatz klar geregelt erschien, fanden die Arzneipflanzen lange in den Entwürfen gar keine Erwähnung. Das wäre das sichere Ende für Neuzulassungen pflanzlicher Tierarzneimittel in der EU gewesen - sie hätten denselben Zulassungsprozess wie Produkte aus Monosubstanzen durchlaufen müssen. Nun findet sich ein einzelner kurzer Doppelsatz im Absatz 12 Präambel der Verordnung (ein deutliches Zeichen für "so grade eben noch in die Verordnung geschafft"): "Bisher liegen keine ausreichenden Informationen über traditionelle pflanzliche Erzeugnisse zur Behandlung von Tieren vor, die die Einführung eines vereinfachten Systems ermöglicht hätten. Daher sollte die Kommission die Möglichkeit der Einführung eines solchen vereinfachten Systems auf der Grundlage der von den Mitgliedstaaten übermittelten Informationen über die Verwendung dieser Erzeugnisse in ihrem Hoheitsgebiet prüfen" [25]. Nun gilt es, Argumente zu finden, die eine vereinfachte Zulassung traditioneller pflanzlicher Tierarzneimittel unterstützen. Bis 2027 bleibt dafür Zeit. 
Nun das wirklich Erfreuliche: Die Schweiz ist in ebendieser Hinsicht einen deutlichen Schritt weiter als die EU. In der seit Anfang 2019 in Kraft getretenen "Verordnung des Schweizerischen Heilmittelinstituts über die vereinfachte Zulassung von Komplementär- und Phytoarzneimittel" (kurz KPAV) [26] heisst es im ersten Artikel: "Gegenstand: Diese Verordnung regelt die Anforderungen, die Komplementär- und Phytoarzneimittel der Humanund Veterinärmedizin erfüllen müssen, und legt die Voraussetzungen für deren vereinfachte Zulassung und die Zulassung im Meldeverfahren fest." Auch hier gibt es noch einiges zu tun, gilt es doch den Begriff der "Tradition", der insbesondere für die vereinfachte Zulassung von Phytoarzneimitteln wichtig ist, mit Leben zu füllen. Anders als in der Humanmedizin fehlen anerkannte tierme- dizinische Monographien und die mindestens 30-jährige Anwendung eines Phytoarzneimittels ist in der Tiermedizin auch wesentlich schwieriger, da es ja seit vielen Jahren kaum mehr zugelassenen Phyto-Tier-Arzneimittel gibt. Ohne Zweifel sollten dies aber überwindbare Hürden sein, denn die ausdrückliche Aufnahme der Veterinärmedizin in den Artikel 1 zeugt ja von der Bereitschaft des Gesetzgebers, Neuzulassungen ermöglichen zu wollen.

Auch für die arzneimittelrechtliche Anerkennung pflanzlicher Tierarzneimittel sind fundierte wissenschaftlich publizierte Erkenntnisse nicht abträglich. Um es abschliessend noch ein letztes Mal zu unterstreichen: Für interessante und qualitativ hochwertige veterinärmedizinische Manuskripte steht die Tür der CMR weit offen.

\section{Literatur}

1 Walkenhorst M. Tiermedizinische Publikationen in Complementary Medicine Research. Complement Med Res. 2017;24(6): 338-41.

2 Bismarck D, Schneider M, Müller E. Antibakterielle In-vitro-Wirksamkeit ätherischer Öle gegen veterinärmedizinisch relevante Keime klinischer Isolate von Hunden, Katzen und Pferden. Complement Med Res. 2017;24(3): 153-63.

3 Biegel U, Stratmann N, Knauf Y, Ruess K, Reif M, Wehrend A. [Postoperative adjuvante Therapie mit einem Mistelextrakt (Viscum album ssp. album) bei Hündinnen mit Mammatumoren] [German.]. Complement Med Res. 2017;24(6):349-57.

4 von Bodungen U, Ruess K, Reif M, Biegel U. [Kombinierte Anwendung von Strahlentherapie und adjuvanter Therapie mit einem Mistelextrakt (Viscum album L.) zur Behandlung des oralen malignen Melanoms beim Hund: eine retrospektive Studie] [German.]. Complement Med Res. 2017;24(6):358-63.

5 Ryhner T, Meier B, Walkenhorst M. [Arzneipflanzen im Berner Pferdestall: Erfahrungswissen von Pferdehaltern]. Complement Med Res. 2018;25(5):331-7.

6 Zhou W, Ouyang J, Wang H, Wang X. Complement Med Res. 2019;26(1):31-8.

7 Maeschli A, Schmidt A, Ammann W, Schurtenberger P, Maurer E, Walkenhorst M. [Einfluss eines komplementärmedizinischen telefonischen Beratungssystems auf den Antibiotikaeinsatz bei Nutztieren in der Schweiz]. Complement Med Res. 2019;26(3):174-81.

8 Journal Club. Complement Med Res. 2017; 24(6):342-8.
9 Abstract Service. Complement Med Res. 2017;24:371-5.

10 Journal Club. Complement Med Res. 2019; 26(3):151-5.

11 Latest Publications You Should Not Miss. Latest Publications You Should Not Miss. Complement Med Res. 2019;26(3):211-2.

12 Stucki K, Cero MD, Vogl CR, Ivemeyer S, Meier B, Maeschli A, et al. Ethnoveterinary contemporary knowledge of farmers in prealpine and alpine regions of the Swiss cantons of Bern and Lucerne compared to ancient and recent literature - Is there a tradition? J Ethnopharmacol. 2019 Apr;234:225-44.

13 Mertenat D, Cero MD, Vogl CR, Ivemeyer S, Meier B, Maeschli A, et al. Ethnoveterinary knowledge of farmers in bilingual regions of Switzerland - is there potential to extend veterinary options to reduce antimicrobial use? J Ethnopharmacol. 2019 Aug;246:112184.

14 Kupper J, Walkenhorst M, Ayrle H, Mevissen M, Demuth D, Naegeli H. Online-Informationssystem für die Phytotherapie bei Tieren. Schweiz Arch Tierheilkd. 2018 Oct;160(10): 589-95.

15 Hardy K. Paleomedicine and the use of plant secondary compounds in the Paleolithic and Early Neolithic. Evol Anthropol. 2019 Mar; 28(2):60-71.

16 Huffman MA. Animal self-medication and ethno-medicine: exploration and exploitation of the medicinal properties of plants. Proc Nutr Soc. 2003 May;62(2):371-81.

17 Provenza FD, Kronberg SL, Gregorini P. Is Grassfed Meat and Dairy Better for Human and Environmental Health? Front Nutr. 2019 Mar 19;6:26.

18 Singer MS, Mace KC, Bernays EA. Self-medication as adaptive plasticity: increased ingestion of plant toxins by parasitized caterpillars. PLoS One. 2009;4(3):e4796.
19 Villalba JJ, Provenza FD. Self-medication and homeostatic behaviour in herbivores: learning about the benefits of nature's pharmacy. Animal. 2007 Oct;1(9):1360-70.

20 Villalba JJ, Provenza FD, Stott R. Rumen distension and contraction influence feed preference by sheep. J Anim Sci. 2009 Jan;87(1): 340-50.

21 Provenza F. (2018). Nourishment: What Animals Can Teach Us about Rediscovering Our Nutritional Wisdom Chelsea Green Publishing ISBN 9781603588027

22 Maßberg D, Hatt H. Human Olfactory Receptors: novel cellular functions outside of the nose. Physiol Rev. 2018 Jul;98(3):1739-63.

23 Ortega Á, Zhulin IB, Krell T. Sensory repertoire of bacterial chemoreceptors. Microbiol Mol Biol Rev. 2017 Oct;81(4):e00033-17.

24 Heusser P. [The Concept of 'Integrative and Personalized Health Care']. Complement Med Res. 2017;24 Suppl 1:1-2.

25 Regulation (EU) 2019/6 of the. European Parliament and of the Council of 11 December 2018 on veterinary medicinal products and repealing Directive 2001/82/EC (https://eurlex.europa.eu/legal-content/EN/TXT/ PDF/?uri=CELEX:32019R0006\&

26 KPAV. 2018. Verordnung des Schweizerischen Heilmittelinstituts über die vereinfachte Zulassung von Komplementär- und Phytoarzneimitteln (Komplementär- und Phytoarzneimittelverordnung) vom 7. September 2018 (Inkrafttreten am 1. Januar 2019); SR 812. 212.24. (https://www.admin. ch/opc/de/classifiedcompilation/20173469/ index.html) 